Mens

revue d'histoire intellectuelle de l'Amérique française

Claude Hauser et Catherine Pomeyrols, dir. L'Action française et l'étranger. Usages, réseaux et représentations de la droite nationaliste française. Paris, L'Harmattan, 2001. 148 p.

\title{
Stéphane St-Pierre
}

Volume 5, numéro 2, printemps 2005

URI : https://id.erudit.org/iderudit/1024370ar

DOI : https://doi.org/10.7202/1024370ar

Aller au sommaire du numéro

Éditeur(s)

Centre de recherche en civilisation canadienne-française

ISSN

1492-8647 (imprimé)

1927-9299 (numérique)

Découvrir la revue

Citer ce compte rendu

St-Pierre, S. (2005). Compte rendu de [Claude Hauser et Catherine Pomeyrols, dir. L'Action française et l'étranger. Usages, réseaux et représentations de la droite nationaliste française. Paris, L’Harmattan, 2001. 148 p.] Mens, 5(2),

532-537. https://doi.org/10.7202/1024370ar d'utilisation que vous pouvez consulter en ligne. 
Claude Hauser et Catherine Pomeyrols, dir. L'Action française et l'étranger. Usages, réseaux et représentations de la droite nationaliste française. Paris, L'Harmattan, 2001. 148 p.

En réalisant l'ouvrage L'Action fransaise et l'étranger, Catherine Pomeyrols, Claude Hauser, Alain Clavien, Emmanuel Hurault, Jacques Prévotat, Denis Rolland et Cécile Vanderpelen tentent de combler un vide historiographique concernant l'influence du maurrassisme à l'extérieur de la France. L'intention précise de ce collectif est d'exposer « comment les intellectuels attirés par l'orbite de l'Action française ont tenté d'acculturer [les] enseignements [de Charles Maurras] dans leurs conceptions de la nation, de l'État et de la société " (p. 5). Or, l'utilisation du maurrassisme « réconcilie droite et intelligence, catholicisme et idées modernes, conservatisme et modernité » en plus de favoriser l'édification d'idéologies de substitution en lieu et place de celles de «la modernité industrielle et laïque » (p. 8). Il est intéressant de constater que l'influence de l'Action française se résume essentiellement à celle de Charles Maurras. Tenant compte de ce fait, il est étonnant de voir les auteurs délaisser l'analyse de la «matrice idéologique » de Maurras pour s'orienter exclusivement vers l'étude des emprunteurs de cette doctrine. En n'effectuant aucune véritable référence à cette «matrice idéologique ", les auteurs s'adressent à ceux qui en possèdent une connaissance approfondie. Cette manière de faire entre en contradiction avec l'intention avouée des auteurs de susciter un intérêt pour cette problématique chez les chercheurs. Il aurait été possible, surtout dans un ouvrage de 148 pages, de présenter une analyse succincte de la doctrine maurrassienne afin de donner plus de cohérence et de profondeur au traitement. Jacques Prévotat aurait été tout indiqué pour mener à bien cette tâche. De plus, l'absence d'une défi- 
nition uniforme et convaincante de ce qu'est un intellectuel maurrassien affaiblit considérablement la comparaison. Jusqu'à quel degré de dilution du maurrassisme, un clerc peut-il être dit maurrassien? Cette question préalable ne reçoit pas de réponse. Ces ajouts auraient favorisé la compréhension de l'évolution des idées maurrassiennes entre la "matrice idéologique » et l'adaptation réalisée par les emprunteurs.

Malgré cette lacune, le lecteur est en mesure de comprendre que les adaptations et les modifications effectuées par les emprunteurs répondent aux besoins spécifiques de leur condition. L'ouvrage met en lumière la facilité avec laquelle il est possible de réinterpréter une idéologie qui s'affiche au départ comme étant " un bloc cohérent et monolithique » ( $p$. 137). Grâce à leur approche comparative des expériences suisse, belge, québécoise, sud-américaine et portugaise, les auteurs ont fait ressortir la flexibilité du processus d'acculturation des idéologies dans différents contextes. Un des chapitres, signé par Serge Rossier, est réservé à l'influence de Maurras sur la pensée du grand écrivain suisse Charles-Albert Cingria. Cette analyse démontre que les idées maurrassiennes ont souvent rayonné par l'entremise de l'esthétique et que les milieux littéraires sont précisément ceux qui prennent le plus de liberté avec leur contenu politique. Les diverses études qui composent ce recueil relèvent deux facteurs favorisant l'enracinement et l'application des idées de Maurras : la présence d'une élite intellectuelle active et responsable et l'expérience d'un événement traumatisant.

On ne peut ici reprendre l'ensemble des hypothèses et des pistes de réflexion proposées concernant la morphologie adoptée par les idées maurrassiennes dans leur diffusion hors de France. Toutefois, les conditions locales, l'héritage historique et la conjoncture politique sont les réalités qui influencent le destin du maurrassisme à l'étranger. À titre d'exemple, 
le maurrassisme vise à affermir et à consolider en Suisse un cadre idéologique fragile et tourmenté. Les contributions de Claude Hauser et d'Alain Clavien offrent des regards complémentaires sur cette question, le second faisant intervenir habilement les effets de génération. Une fois adaptées au contexte politique et social, les idées maurrassiennes deviennent des outils d'affirmation nationale, non sans alimenter des débats parfois fratricides. Pourtant, il est légitime de se demander si certains auteurs de ce collectif n'ont pas été obnubilés par leur volonté d'établir à tout prix le retentissement du maurrassisme sur l'évolution des idéologies nationales. Ceuxci semblent conférer, dans certaines analyses moins réussies, une influence et un rôle exagérés aux idées maurrassiennes alors que leur démonstration ne justifie pas un tel constat. Encore une fois, l'on ne parvient pas toujours à distinguer entre assimilation, contamination et convergence.

L'un des aspects les plus innovateurs de ce collectif réside dans l'analyse des relais et des réseaux par lesquels s'infiltre, se diffuse et s'affirme l'influence de l'école maurrassienne. Par l'entremise de ces réseaux, les idées sont remaniées, réutilisées et transformées. Selon Denis Rolland en Amérique latine, il arrive que l'influence soit indirecte et qu'il s'agisse d'un Maurras transformé et adapté par les lecteurs espagnols et les sympathisants du franquisme et du salazarisme. Le Maurras proposé n'est plus celui de l'origine, mais celui répondant aux besoins spécifiques des Espagnols et des Portugais et qui sera par la suite réadapté aux besoins des Latino-Américains. Toutefois, il est difficile alors de cerner l'influence réelle du maurrassisme puisque cette doctrine est masquée par son imbrication à d'autres idéologies, ce qui la rend moins perceptible. Denis Rolland a donc raison de multiplier les précautions. Néanmoins, selon lui, la doctrine de Charles Maurras a eu une influence en Amérique latine, en 
particulier sur le «traditionalisme catholique latino-américain et certaines références idéologiques des gouvernements autoritaires de la région" (p.122). Les idées maurrassiennes deviennent ainsi "l'outil d'une stratégie politique et intellectuelle au service d'un groupe à vocation prosélytique » (p. 9).

La condamnation pontificale de l'Action française en 1926 est un autre thème abordé dans le cadre de ce collectif. Selon Jacques Prévotat, cette condamnation révèle l'attachement d'un certain nombre d'intellectuels à un système ou à une doctrine qui comble des manques ou des désarrois. En Belgique, le maurrassisme devient une arme de la bourgeoisie francophone dans sa lutte contre le flamingantisme. Selon Cécile Vanderpelen, qui relativise les effets de la condamnation, cet intérêt pour le maurrassisme trahit une certaine incapacité du catholicisme belge à penser un système idéologique audacieux et mobilisateur en ne puisant que dans son propre fonds. Il est intéressant de relever une astuce de certains catholiques belges : "la double appartenance de Maurras aux sphères politique et littéraire permet justement à ses disciples de prétendre éprouver pour son œuvre un intérêt purement esthétique, exempt de préoccupation politique et, ainsi, espérer échapper à la condamnation » (p. 70).

C'est en tenant compte du contexte de la condamnation pontificale de 1926 que Catherine Pomeyrols essaie d'exposer la crise entourant l'Action française au Québec. Selon elle, c'est à la faveur de la crise que Henri Bourassa prêche " un accord entre les catholiques de toutes races » (p. 81). La position qu'il défendait depuis 1922 est consolidée alors que celle de Groulx, nationaliste de l'Action française, est fragilisée. Le catholicisme ultramontain et le nationalisme pancanadien de Bourassa se trouvent ainsi "accordés ", sans qu'on sache trop où se situait la contradiction. Cette crise aurait mené les publications catholiques au Québec à recti- 
fier leur position en les forçant à la prudence. Malheureusement, cette analyse de Pomeyrols déçoit par son manque de profondeur - seulement treize pages y sont consacrées - et ses affirmations peu étayées. Il aurait été nécessaire de montrer les contorsions et les contraintes auxquelles l'Action française de Montréal a été soumise, parait-il, au cours de cette crise. Aussi, il aurait été utile d'exposer les divergences autant que les accords entre les conceptions de Lionel Groulx et celles de Charles Maurras, en ce qui a trait, par exemple, à la définition de la nation. Le récit de la crise de la condamnation au Québec y aurait gagné en vraisemblance. Le sens de la nuance de Cécile Vanderpelen aurait fait merveille ici.

Pour sa part, Emmanuel Hurault inverse les perspectives en analysant les répercussions du salazarisme sur les maurrassiens. En fait, Salazar devient, pour les fidèles de Maurras et pour Maurras lui-même, « un modèle de vertus et de conscience du bien commun » (p. 123). Ce régime incarne le modèle maurrassien à sa façon - c'est-à-dire sàns monarchie, ce qui constitue un écart qui est loin d'être insignifiant - et fait ainsi la preuve, aux yeux des maurrassiens, que leurs « idées sont et doivent être mises en œuvre» (p. 132).

En définitive, malgré certaines pistes de réflexions intéressantes, l'analyse réalisée par la plupart des auteurs est décevante. Les hypothèses, abondantes, sont fragiles et peu convaincantes. La démonstration et l'argumentation manquent de rigueur. Les conclusions sont partielles et précipitées. D'ailleurs, c'est principalement sur une base hypothétique que l'argumentation est assez souvent appuyée. Les auteurs n'ont pas été en mesure de surmonter l'obstacle de la cohérence, problème auquel est confrontée la majorité des ouvrages collectifs. Ils vont de l'analyse des adaptations des idées maurrassiennes aux réseaux et aux relais, en passant par l'étude des échos de la condamnation pontificale, sans réussir à vrai- 
ment justifier l'inclusion de ce dernier problème. Dans ce cheminement, le lecteur a du mal à saisir l'évolution et la fécondité de la comparaison. En fait, les liens entre les chapitres sont déficients ou inexistants. Heureusement, Jacques Prévotat parvient, en conclusion, à donner un certain sens à cet ouvrage en complétant et en redressant parfois, sans y paraître, les propos de Claude Hauser et de Catherine Pomeyrols dans l'introduction. On est frappé de constater que cet ouvrage ne présente aucune orientation bibliographique. Ce fait est particulièrement étonnant lorsque l'on sait que l'un des objectifs des auteurs est d'attirer les chercheurs vers ces questions. Dans cette optique, on aurait aimé être renseigné sur l'état actuel des recherches. Le travail d'édition a été bâclé. La présentation biographique des collaborateurs en oublie deux sur huit. Le contenu de la note 64 (p. 114) a été reporté au texte (p. 104), sans qu'on pense à la supprimer. Oublions les coquilles. Néanmoins, malgré ces nombreuses lacunes, cet ouvrage introduit des pistes de réflexion sur un sujet méconnu et peu exploité.

Stéphane St-Pierre

Département d'bistoire

Université de Montréal

\section{Yvan Lamonde et Sophie Montreuil, dir. Lire au Québec au $\boldsymbol{X I X}^{\mathrm{e}}$ siècle. Montréal, Fides, 2003. 330 \\ p.}

Tous ceux que passionne l'histoire culturelle se réjouiront de la sortie de cet ouvrage. L'histoire du livre et de la lecture, dont Yvan Lamonde a été un des éminents pionniers au Québec, se trouve ici enrichie d'une série d'études classées en deux parties dans le présent ouvrage: "Pratiques in- 